\title{
Isolation and structural characterization of a cDNA clone encoding the human DNA repair protein for $O^{6}$-alkylguanine
}

\author{
( $O^{6}$-methylguanine-DNA methyltransferase/ada/MGMT gene)
}

\author{
Keizo Tano*, Susumu Shiota*, Julia Collier*, Robert S. Foote*, and Sankar Mitra ${ }^{\dagger}$ \\ ${ }^{*}$ The University of Tennessee-Oak Ridge Graduate School of Biomedical Sciences and ${ }^{\dagger}$ Biology Division, Oak Ridge National Laboratory, Oak Ridge, \\ TN 37831 \\ Communicated by William L. Russell, October 31, 1989 (received for review September 23, 1989)
}

\begin{abstract}
O^{6}$-Methylguanine-DNA methyltransferase (MGMT; DNA- $O^{6}$-methylguanine:protein-L-cysteine $S$-methyltransferase, EC 2.1.1.63), a unique DNA repair protein present in most organisms, removes the carcinogenic and mutagenic adduct $O^{6}$-alkylguanine from DNA by stoichiometrically accepting the alkyl group on a cysteine residue in a suicide reaction. The mammalian protein is highly regulated in both somatic and germ-line cells. In addition, the toxicity of certain alkylating drugs in tumor and normal cells is inversely related to the levels of this protein. The cDNA of the human gene, henceforth named MGMT, has been cloned in an expression vector on the basis of its rescue of a methyltransferasedeficient $\left(\mathrm{ada}^{-}\right)$Escherichia coli host. A 22-kDa active methyltransferase encoded entirely by the cDNA contains an amino acid sequence of 61 residues that bears $60-65 \%$ similarity with segments of $\boldsymbol{E}$. coli methyltransferase (products of the $a d a$ and ogt genes), which encompass the alkyl-acceptor residues. The human cDNA has no sequence similarity with the $a d a$ and ogt genes, due in part to differences in codon usage, and shows no detectable homology with $\boldsymbol{E}$. coli genomic DNA. However, it hybridizes with distinct restriction fragments of human, mouse, and rat DNAs. The lack of methyltransferase observed in many human cell lines is due to the absence of the $M G M T$ gene or to lack of synthesis and/or stability of its 0.95 -kilobase poly $(\mathbf{A})^{+}$RNA transcript.
\end{abstract}

$O^{6}$-Alkylguanine is the major mutagenic and carcinogenic lesion in DNA induced by simple alkylating mutagens because of its preference for pairing with thymine during DNA replication $(1,2)$. This adduct in DNA is removed by a ubiquitous and unique repair protein, $O^{6}$-methylguanineDNA methyltransferase (MGMT; DNA- $\mathrm{O}^{6}$-methylguanine: protein-L-cysteine $S$-methyltransferase, EC 2.1.1.63), present in most species. This protein, unlike true enzymes, accepts the alkyl group from the lesion in a stoichiometric second-order reaction (3-5). The methyl-acceptor residue in all cases tested so far is cysteine. The major MGMT in Escherichia coli is the inducible $39-\mathrm{kDa}$ product of the ada gene (Ada protein), a multifunctional protein that positively regulates its own and other alkylation repair genes $(6,7) . E$. coli has another, noninducible, MGMT, the 19-kDa Ogt protein (8).

In contrast to the Ada protein, human and rodent MGMTs $(18-24 \mathrm{kDa})$ function solely as alkyl acceptors for $O^{6}$ alkylguanine (9). While mammalian MGMT is not induced as a specific response to DNA alkylation as seen in $E$. coli (10), its expression is highly regulated in different tissues and cell lines and may be heterogeneous within the same population. A number of factors, including tissue, cell type, age, and cell cycle, appear to regulate its level both in vivo and in vitro (11-13). The mutagenic response for $N$-ethyl- $N$-nitrosourea,

The publication costs of this article were defrayed in part by page charge payment. This article must therefore be hereby marked "advertisement" in accordance with 18 U.S.C. $\$ 1734$ solely to indicate this fact. one of the most potent mutagens in the mouse, suggests regulation of MGMT during germ-cell differentiation (14). MGMT activity is undetectable ( $<200$ molecules per cell) in certain cell lines called $\mathrm{Mex}^{-}$or $\operatorname{Mer}^{-}(10,15,16)$. Some subclones of human HeLa S cells-e.g., one obtained from the Massachusetts Institute of Technology Cell Culture Center (HeLa M) - are $\mathrm{Mex}^{-}$, while another subclone-obtained from the American Type Culture Collection (HeLa S3)-has the highest level of MGMT $\left(1.8 \times 10^{5}\right.$ molecules per cell $)$ among all mammalian lines tested $(10,17)$. $M G M T$ expression, while predictable in human/human somatic cell hybrids, appears to be controlled by multiple genetic and epigenetic factors in $\mathrm{Mex}^{+}$human-Mex ${ }^{-}$rodent hybrid cells (17-19). Transformation of $\mathrm{Mer}^{+}$human cells with simian virus 40 frequently induces the $\mathrm{Mer}^{-}$phenotype, which is observed most commonly in tumor lines $(9,15,18)$. On the other hand, certain tumors have been found to be resistant to alkylating drugs such as procarbazine and chloroethyl$N$-nitrosoureas, and this resistance can be correlated with their elevated levels of MGMT $(20,21)$. Thus, an understanding of the basis of MGMT regulation could lead to improvement in cancer chemotherapy.

To identify the factors that regulate MGMT activity, we initiated a program to clone the human $M G M T$ gene $\ddagger$ and its cDNA. Previous attempts to clone this gene by its retrieval from $\mathrm{Mex}^{-}$rodent cells transfected with human DNA have been unsuccessful $(22,23)$. In devising an alternative strategy, we reasoned that, because of the similar biochemical properties of human and $E$. coli MGMTs, it might be possible to clone the human MGMT cDNA from an expression library by phenotypic rescue of $a d^{-} E$. coli on the basis of its increased resistance to an alkylating agent. This report describes the isolation and characterization of such a cDNA clone with this approach. $\S$ The availability of the cDNA clone now provides the opportunity for cloning the human and rodent $M G M T$ genes and elucidating the mechanisms of their regulation.

\section{MATERIALS AND METHODS}

Bacterial Strains and Plasmids. E. coli GW7101 with a deletion in ada ( $\Delta a d a-25)$ was derived from AB1157 (24). JM107 (25), which carries $\operatorname{lacl}^{q}$, was used for expression of human MGMT. The construction of pSM41, a high expression plasmid for Ada protein, has been described earlier (26).

Culture of Human Cell Lines. HeLa S3, used previously (10), and HeLa $M$ were grown in suspension cultures in

Abbreviations: MGMT, $O^{6}$-methylguanine-DNA methyltransferase; MNNG, $N$-methyl- $N$-nitro- $N$-nitrosoguanidine; CNU, 2-chloroethyl- $N$-nitrosourea.

₹The designation has been approved by the Human Gene Mapping Nomenclature Committee.

$\S$ The sequence reported in this paper has been deposited in the GenBank data base (accession no. M29971). 
minimal essential medium modified for suspension culture (S-MEM) containing 5\% (vol/vol) fetal calf serum. The $\mathrm{Mex}^{-}$ lymphoblastoid L33 cells, obtained from B. Strauss (University of Chicago) (19), were grown in RPMI 1640 medium containing $15 \%$ fetal calf serum.

Construction of a cDNA Library. A 5- $\mu$ g aliquot of poly(A) ${ }^{+}$ RNA, purified from $1 \times 10^{8} \mathrm{HeLa} \mathrm{S3}$ cells by two successive passages through an oligo(dT)-cellulose column (27), was used for the synthesis of double-stranded cDNA (28) with a cDNA synthesis kit as recommended by the supplier (Pharmacia). After addition of EcoRI adaptors, the cDNA was ligated to pUC9 DNA (25) that had been linearized with EcoRI and treated with calf intestinal phosphatase (27). Competent $E$. coli DH5 $\alpha$ cells (Bethesda Research Laboratories) were transformed with the cDNA library, yielding $5 \times$ $10^{5}$ independent transformants. After amplification, the total plasmid pool was extracted and purified (27).

Southern Blot Analysis. Fifteen micrograms of EcoRIdigested DNA was separated by electrophoresis in $0.8 \%$ agarose and transferred to a Nylon membrane (GeneScreenPlus, DuPont) for hybridization with the cDNA insert of pKT100 labeled with $\left[\alpha-{ }^{32}\right.$ P $]$ dCTP by the random hexanucleotide priming method (29). The hybridization was carried out at $42^{\circ} \mathrm{C}$ for $24 \mathrm{hr}$ in $3 \times \operatorname{SSPE}(1 \times=0.15 \mathrm{M} \mathrm{NaCl} / 0.1 \mathrm{M}$ sodium phosphate/1 mM EDTA, pH 7.4) containing $1 \times$ Denhardt's solution (27), $10 \%$ dextran sulfate, $1 \%$ sodium dodecyl sulfate (SDS), $49 \%$ formamide, and $0.5 \mathrm{mg}$ of heatdenatured salmon sperm DNA per ml. The filter was washed with $0.03 \mathrm{M} \mathrm{NaCl} / 0.003 \mathrm{M}$ sodium citrate, $\mathrm{pH} \mathrm{7/0.1 \%} \mathrm{SDS}$ at $65^{\circ} \mathrm{C}$ for $1 \mathrm{hr}$, dried, and exposed to x-ray film.

RNA (Northern) Blot Analysis. Two micrograms of poly(A) ${ }^{+}$RNA purified by chromatography on oligo(dT)cellulose was electrophoresed in $1.2 \%$ agarose containing 2.2 $\mathbf{M}$ formaldehyde (27) and transferred to a Nylon membrane (Hybond-N, Amersham). Hybridization with the ${ }^{32} \mathrm{P}$-labeled cDNA insert of pKT100 was carried out at $65^{\circ} \mathrm{C}$ in $1 \%$ nonfat dry milk/2 mM EDTA/7\% SDS in $500 \mathrm{mM}$ sodium phosphate (pH 7.2) for about $24 \mathrm{hr}$. The filters were washed with $0.15 \mathrm{M}$ $\mathrm{NaCl} / 0.015 \mathrm{M}$ sodium citrate, $\mathrm{pH} 7 / 0.1 \% \mathrm{SDS}$ at $65^{\circ} \mathrm{C}$ for 1 $\mathrm{hr}$, dried, and exposed to X-ray film.

Assay of Methyltransferase. Synthetic poly $\left(\mathrm{dC}, \mathrm{dG},\left[8-{ }^{3} \mathrm{H}\right]-\right.$ $\mathrm{m}^{6} \mathrm{dG}$ ) was used as the substrate for the MGMT assay as described (10). The growth of the $E$. coli cells and preparation of crude extracts used in these assays have been described (26).

Nucleotide Sequence Analysis. The 835-base-pair (bp) cDNA insert fragment of pKT100 was sequenced by the chain-termination method $(30,31)$ with Sequenase version 2.0 (United States Biochemical) and 17- to 20-base-long oligonucleotide primers. DNA sequence analysis and the comparison of amino acid sequences were performed by using the DNASIS (Pharmacia) and Pustell Sequence Analysis Programs (IBI) and the National Biomedical Research Foundation Protein Identification Resource data base software (release no. 59, June 1989) on an NCR personal computer.

\section{RESULTS}

Cloning of cDNA of Human MGMT. The increased resistance of ada $^{-} E$. coli cells containing active human MGMT to alkylating agents was used as the basis for cloning the MGMT cDNA from a HeLa S3 expression library. We chose HeLa S3 cells because of their high level of MGMT (10) and presumably high level of MGMT poly $(A)^{+}$RNA. The $E$. coli GW7101 host used for screening the library has a deletion in ada that prevents its reversion to $\mathrm{ada}^{+}$during exposure to the alkylating mutagen. Control experiments with GW7101 and its ad $^{+}$parent, AB1157, showed a significant difference in their sensitivity to $N$-methyl- $N^{\prime}$-nitro- $N$-nitrosoguanidine (MNNG) (24). GW7101 cells, transformed with the cDNA library, were suspended at $5 \times 10^{6}$ cells per $\mathrm{ml}$ in $0.9 \% \mathrm{NaCl}$ and exposed to $100 \mu \mathrm{g}$ of MNNG per ml for $30 \mathrm{~min}$ at $37^{\circ} \mathrm{C}$. The cells were then plated on LB agar (27) with ampicillin. Cells from surviving colonies were harvested and resuspended in $0.9 \% \mathrm{NaCl}$, and the procedure was repeated. Single colonies of cells that survived after three cycles of MNNG treatment were found to be more resistant to MNNG ( $90 \%$ survival) than the starting population (10\% survival). From these cells two populations of plasmids were isolated containing inserts of about 250 and $800 \mathrm{bp}$, respectively. Retransformation of GW7101 with the individual plasmids showed that only the one with the larger insert (henceforth called pKT100) conferred MNNG resistance.

Activity of MGMT Encoded by Human cDNA. Table 1 shows MGMT activity in $E$. coli coded by pKT100 and the control plasmids. The trace MGMT activity in GW7101 (pUC9) was presumably due to the Ogt protein. E. coli JM107 $\left(a d a^{+}\right)$has a significant, basal level of MGMT. Isopropyl $\beta$-D-thiogalactoside induction of MGMT coded by pKT100 in lacI $^{q}$-containing $\mathrm{JM} 107$ indicates that MGMT expression from the plasmid is under lac promoter control. To test whether human MGMT was produced from pKT100 as a fusion polypeptide with the $\beta$-galactosidase leader sequence (25), the plasmid pKT110 was constructed by blunt-end ligation of BamHI-linearized pKT100 after the sticky ends were filled by DNA polymerase I Klenow fragment (27). The addition of four nucleotides altered the reading frame in the lac $Z$ sequence $5^{\prime}$ upstream of the cDNA sequence in the plasmid. The MGMT level expressed from pKT110 was comparable to that of pKT100, which was $1 / 5$ th to $1 / 20$ th of the activity coded by the high expression $E$. coli ada plasmid pSM41 (26).

Nucleotide Sequence of Human MGMT. Fig. 1 shows the nucleotide sequence of the MGMT cDNA. The presence of a termination codon (TAG) at position 26 would prevent synthesis of a fusion protein of MGMT and the $\beta$ galactosidase leader sequence from the vector. A computer search of the open reading frames in the cDNA sequence showed several possibilities, only two of which, with initiation codons at positions 97 and 115 , could code for a 20 - to 22-kDa polypeptide. MGMT was purified to near homogeneity from isopropyl $\beta$-D-thiogalactoside-induced JM107 (pKT100) and was found to have a size of about $22 \mathrm{kDa}$ (unpublished data). About $20 \mu \mathrm{g}$ of the protein was subjected to SDS/PAGE and transferred to Immobilon Transfer membrane (Millipore) (32) for $\mathrm{N}$-terminal sequence analysis in a gas-phase sequencer (Applied Biosystems model 470A). The phenylthiohydantoin-conjugated amino acids were identified by HPLC (33). The sequence determined, $\mathrm{NH}_{2}$-MetAsp-Lys-Asp-Xaa-Glu-Met-Lys, uniquely placed the initiation codon at position 97 . It is important to note that the second ATG codon at position 115 is in the same reading frame as the ATG at position 97, with the termination codon for both being at position 718. The MGMT, based on initiation at position 97, has a predicted molecular weight of 21,645 , in excellent agreement with the observed value.

Table 1. MGMT activity encoded by human cDNA

\begin{tabular}{clcc}
\hline E. coli host & Plasmid & Treatment & $\begin{array}{c}\text { Specific activity, } \\
\text { pmol/ } \mu \text { g of protein }\end{array}$ \\
\hline GW7101 & pUC9 & None & 0.07 \\
& pKT100 & None & 10.4 \\
JM107 & pUC9 & None & 0.71 \\
& & IPTG & 0.62 \\
& pKT100 & None & 26.2 \\
& & IPTG & 86.0 \\
& pKT110 & IPTG & 57.3 \\
& pSM41 & IPTG & $449^{*}$ \\
\hline
\end{tabular}

IPTG, isopropyl $\beta$-D-thiogalactoside.

*This value, originally shown in ref. 26 , is included for comparison. 
1 ATG GAC AAG GAT TGT GAA ATG AAA CGC ACC ACA CTG GAC AGC CCT TTG Met Asp Lys Asp Cys Glu Met Lys Arg Thr Thr Leu Asp Ser Pro Leu 10

145 GGG AMG CTG GAG CTG TCT GGT TGT GAG CAG GGT CTG CAC GAA ATA AAG Gly Lys Leu Glu Leu Ser Gly Cys Glu Gln Gly Leu His Glu Ile Lys 20

193 CTC CTG GGC AAG GGG ACG TCT GCA GCT GAT GCC GTG GAG GTC CCA GCC Lou Lou Gly Lys Gly Thr Ser Ala Ala Asp Ala Val Glu Val Pro Ala 40

241 CCC GCT GCG GTT CTC GGA GGT CCG GAG CCC CTG ATG CAG TGC ACA GCC Pro Ala Ala Val Lou Gly Gly Pro Glu Pro Leu Met Gln Cys Thr Ala so 60

289 TGG CTG AAT GCC TAT TTC CAC CAG CCC GAG GCT ATC GAA GAG TTC CCC Trp Leu Asn Ala Tyr Phe His Gln Pro glu Ala Ile Glu Glu Phe Pro 70

337 GTG CCG GCT CTT CAC CAT CCC GTT TTC CAG CAA GAG TCG TTC ACC AGA Val Pro Ala Leu His His Pro Val Phe Gln Gln Glu Ser Phe Thr Arg 90

385 CAG GTG TTA TGG AAG CTG CTG AAG GTT GTG AAA TTC GGA GAA GTG ATT Gln Val Leu Trp Lys Leu Leu Lys Val Val Lys Phe Gly Glu Val Ile 100 110

433 TCT TAC CAG CAN TTA GCA GCC CTG GCA GGC AAC CCC AAA GCC GCG CGA Ser Tyr Gln Gln Leu Ala Ala Leu Ala Gly Asn Pro Lys Ala Ala Arg 120

481 GCA GTG GGA GGA GCA ATG AGA GGC AAT CCT GTC CCC ATC CTC ATC CCC Ala Val Gly Gly Ala Met Arg Gly Asn Pro Val Pro Ile Leu Ile Pro 130 Gro 140

529 TGC CAC AGA GTG GTC TGC AGC AGC GGA GCC GTG GGC AAC TAC TCC GGA Cys His Arg Val Val Cys Ser Ser Gly Ala Val Gly Asn Tyr Ser Gly 150

577 GGA CTG GCC GTG AAG GAA TGG CTT CTG GCC CAT GAA GGC CAC CGG 160 Gly Leu Ala Val Lys Glu Trp Leu Leu Ala His Glu Gly His Arg Leu 170

625 GGG AAG CCA GGC TTG GGA GGG AGC TCA GGT CTG GCA GGG GCC TGG CTC Gly Lys Pro Gly Leu Gly Gly Ser Ser Gly Leu Ala Gly Ala Trp Leu 180 190

673 AAG GGA GCG GGA GCT ACC TCG GGC TCC CCG CCT GCT GGC CGA AAC TGA Lys Gly Ala Gly Ala Thr Ser Gly Ser Pro Pro Ala Gly Arg Asn End 200

721 GTATGTGCAGTAGGATGGATGTTTGAGCGACACACACGTGTAACACTGCATCGGATGCGGGGC

784 GTGGAGGCACCGCTGTATTAAAGGAAGTGGCAGTGTCCCTCGTGCCGAATTC

FIG. 1. Nucleotide sequence of the cDNA clone of human MGMT with the deduced amino acid sequence.

Sequence Similarity Among Human and $E$. coli MGMTs. A comparison of the amino acid sequence of the human methyltransferase with those of the Ada and Ogt proteins of $E$. coli $(6,8)$ shows some identical residues scattered along the whole molecules. (Only the C-terminal half of the Ada protein containing the MGMT active site was used for comparison.) However, a remarkably similar sequence is observed among amino acid residues 285-345 of Ada protein, 103-163 of Ogt protein, and 109-169 of the human protein (Fig. 2). Among the 61 residues, 25 are identical in all three proteins. Thirtysix residues of the Ada protein and 40 residues of the Ogt protein are identical or similar to the residues of human MGMT in this stretch. These sequences include the alkylacceptor residues Cys-321 of Ada and Cys-139 of Ogt proteins. Four residues bracketing the cysteine in the segment Pro-Cys-His-Arg-Val are identical in all three proteins. We may reasonably predict from this sequence similarity that Cys-145 of human MGMT is the alkyl-acceptor for $O^{6}$. alkylguanine in DNA.

A similarity search, based on the FASTP program (34), of the translations of MGMT cDNA against those of 15,946 sequences in GenBank showed that only $E$. coli Ada and Ogt proteins have a significant similarity with the human MGMT.

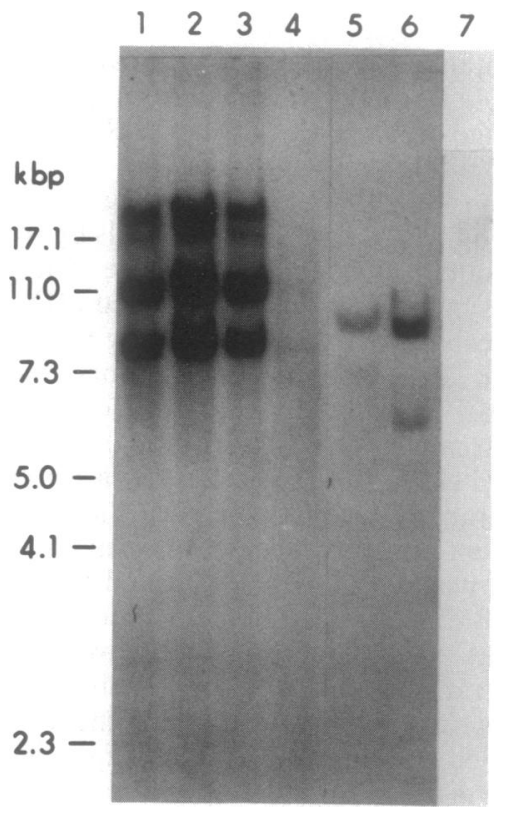

Fig. 3. Southern blot analysis of EcoRI-digested DNAs from human placenta (lane 1), $\mathrm{Mex}^{+} \mathrm{HeLa}$ S3 (lane 2), $\mathrm{Mex}^{-} \mathrm{L} 33$ (lane 3), Mex- HeLa M (lane 4), rat F344 spleen (lane 5), mouse BALB/c liver (lane 6), and $E$. coli AB1157 (lane 7) with ${ }^{32}$ P-labeled human MGMT cDNA. The positions of HindIII-digested T5 DNA fragment markers (35) are indicated. kbp, Kilobase pairs.

With an average score of 21.6 and a standard deviation of 7.5, the scores for $a d a$ and ogt genes were 214 and 248, respectively. As already indicated, most of the sequence identity was observed in the region around the alkyl-acceptor cysteine. Pig mitochondrial malate dehydrogenase was the only other interesting entry whose score of 87 was comparable to that of many other obviously dissimilar proteins. However, it has the same Ile-Pro-Cys-His sequence of the active site of methyltransferase. The significance of this sequence identity is not clear.

Analysis of MGMT Gene Sequences in Human Cells. Southern-blot analysis of genomic DNAs from human cells shows that fragments of identical size-namely, about $23,10.5$, and 7.5 kilobases $(\mathrm{kb})$ in EcoRI-digested DNAs from human placenta, Mex ${ }^{+} \mathrm{HeLa} \mathrm{S} 3$, and $\mathrm{Mex}^{-}$L33 cells hybridized with the ${ }^{32} \mathrm{P}$-labeled cDNA of pKT100 (Fig. 3). In contrast, DNA from $\mathrm{Mex}^{-} \mathrm{HeLa} \mathrm{M}$ cells did not hybridize. Fig. 3 also shows that rat and mouse DNAs contain similar but not identical sequences to the human $M G M T$ gene and that $E$. coli DNA did not hybridize to a detectable extent with human cDNA.

Analysis of Human MGMT Transcript. Northern blot analysis of poly $(\mathrm{A})^{+}$RNAs from human cells shows the presence of a single 950-nucleotide-long human MGMT transcript in HeLa S3 cells (Fig. 4A). No hybridizable band was detected in the poly(A) ${ }^{+}$RNAs of Mex ${ }^{-}$HeLa M and L33 cells. All three lines had comparable levels of $\gamma$-actin message as observed by hybridization of its cDNA probe with single

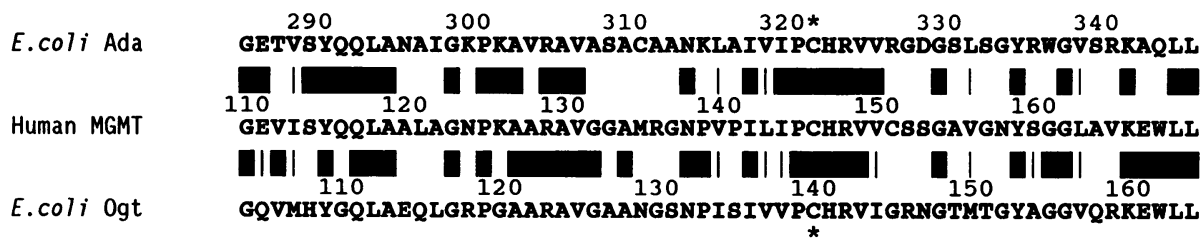

FIG. 2. Sequence similarity of human and $E$. coli methyltransferases. Amino acid residues 109-169 of human MGMT, 285-345 of $E$. coli Ada protein (6), and 103-163 of Ogt protein (8) are aligned for maximum fit. The identical amino acids (denoted by single letter codes) are marked by boxes, and similar ones are marked by lines. The alkyl-acceptor cysteines of Ada and Ogt are indicated by asterisks. 


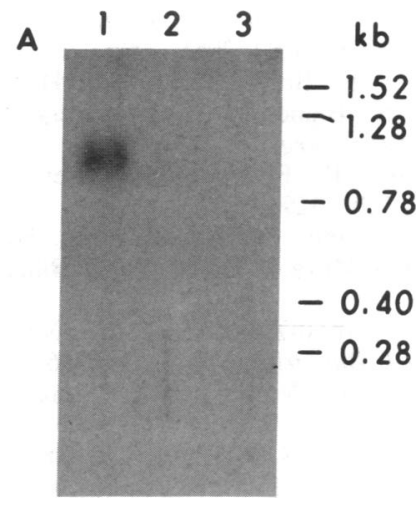

B

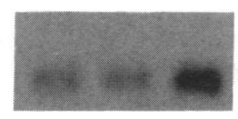

FIG. 4. (A) Northern blot analysis of poly $(A)^{+}$RNAs from $\mathrm{Mex}^{+}$ HeLa S3 (lane 1), Mex- HeLa M (lane 2), and Mex- L33 (lane 3) with ${ }^{32}$ P-labeled human MGMT cDNA. (B) The same filter probed with ${ }^{32} \mathrm{P}$-labeled cDNA of human $\gamma$-actin (36).

bands on the same blot (Fig. $4 B$ ), thus confirming the integrity of the poly $(A)^{+}$RNAs from each of the cell lines.

\section{DISCUSSION}

This report documents the first isolation and characterization of the cDNA of a human repair gene, $M G M T$, which is highly regulated and whose gene product has been biochemically well characterized. In view of the essential role of DNA repair processes in all organisms and the regulation of these activities, it is surprising that, although the biochemical and phenomenological studies of mammalian DNA repair have been extensive, very little is known about the molecular mechanisms of their regulation. In fact, only two other mammalian DNA repair genes, both involved in excision repair, have been cloned so far $(37,38)$. The biochemical functions of their gene products, which are yet to be isolated, remain unknown. In contrast to excision repair proteins, which act in a complex fashion, those involved in removing alkyl adducts in DNA act singly and thus allow a straightforward approach to the cloning of their genes. Because these latter repair processes are quite similar in $E$. coli and mammals and because the repair mutants of $E$. coli have been well characterized, we speculated that repair-deficient $E$. coli mutants could be rescued by analogous mammalian repair proteins expressed from plasmids in these cells. The obvious requirement for such an approach in cloning mammalian cDNAs is that the mammalian protein can function effectively in the intracellular environment of $E$. coli. The major advantage of this approach is that an active repair protein is synthesized in $E$. coli and can be purified. Based on earlier biochemical studies of human MGMT, we believed that it would be a good candidate for this approach. The use of $E$. coli mutants deficient in distinct repair enzymes has enabled us to clone not only the cDNA of human MGMT, but also the cDNA of human $N$-methylpurine-DNA glycosylase from the same cDNA expression library (D. Chakravarti and S.M., unpublished data).

The polypeptides synthesized from cloned cDNAs under lac promoter control of pUC expression vectors are expected to be fusion products with their $\mathrm{N}$-terminal leader peptides encoded by the lac $Z$ gene segments of the vectors themselves. To test whether this was indeed the case with the MGMT cDNA, pKT100 was modified to pKT110 in which the reading frame of the lac $Z$ sequence was changed. The fact that this modification did not cause a loss of MGMT suggested that only one of these plasmids directed synthesis of an MGMT fusion protein. Thus, the complete coding sequence of active MGMT was contained in the cDNA. After determining the nucleotide sequence of the cDNA, we found several open reading frames, only two of which, in the same phase, could code for the 21- to $22-\mathrm{kDa}$ MGMT polypeptide that we have purified to near homogeneity. The sequence of the first eight amino acids at the $\mathbf{N}$ terminus of purified MGMT encoded by pKT100 identified the location of the initiation codon. It appears that the ribosome-binding sequence needed for translation in $E$. coli (possibly GGA six nucleotides upstream of the start codon) is fortuitously present in the MGMT cDNA.

The MGMT poly(A) ${ }^{+}$RNA of about 950 nucleotides is longer than the cDNA by more than 100 nucleotides. In the absence of a poly(A) stretch as well as the consensus polyadenylylation signal sequence (AATAAA) expected to be present at the $3^{\prime}$ end of the MGMT message but lost in the cDNA insert, we do not know how many more untranslated nucleotides are missing at the $3^{\prime}$ end of the cDNA, although the termination codon of the protein was preserved. It is also possible that some of the $5^{\prime}$ nucleotide sequences of the poly $(\mathrm{A})^{+}$RNA were lost during the synthesis of the cDNA, including the indigenous ATG start codon. If so, some of the $\mathrm{N}$-terminal amino acids are not essential for the MGMT activity.

The high degree of sequence similarity between the alkylacceptor regions of $E$. coli MGMTs and a comparable region of the human protein may be an example of common ancestry of important bacterial and mammalian proteins or a remarkable example of convergent evolution (39). However, there is little similarity in the coding sequence of the human MGMT with that of the $E$. coli proteins in this region because of differences in codon usage. In contrast and not surprisingly, the coding sequences among the human and rodent $M G M T$ genes are conserved (Fig. 3).

One of our major objectives in cloning the human MGMT cDNA is to investigate the biochemical basis for the absence of MGMT in $\mathrm{Mex}^{-}$human cell lines. The lack of the protein could be the result of the loss of its gene or a lack of synthesis and/or stability of its transcript. Northern blot analysis of two such $\mathrm{Mex}^{-}$cell lines (HeLa M and L33) showed, as expected, that these cells do not contain detectable MGMT poly $(\mathrm{A})^{+}$RNA. Interestingly, in HeLa M, the MGMT DNA sequence was missing as determined by Southern blot analysis, while L33 lymphoblastoid cells appeared to have an unaltered MGMT gene. Thus, at least in some $\mathrm{Mex}^{-}$cells, the $M G M T$ gene is either not transcribed or the message is unstable. The various genetic and epigenetic factors that control MGMT transcription in human and rodent cells can now be studied with the isolation of genomic clones.

We thank W. C. Dunn and K. R. Isham for technical help and K.-L. Lee for helpful discussions. G. C. Walker and B. Strauss kindly provided $E$. coli GW7101 and L33 cell line, respectively. C. Stringer in F. C. Hartman's laboratory carried out the experiments on N-terminal sequencing of the methyltransferase. L.-Y. Chang kindly provided the $\gamma$-actin probe. We acknowledge A. Makkinje's kind help in the use of DNASIS software. We also thank F. W. Larimer for carrying out the analysis of amino acids and nucleotide sequences of the GenBank. This work was supported by National Cancer Institute Grant CA 31721 and by the U.S. Department of Energy under Contract DE-ACO5-84OR21400 with Martin Marietta Energy Systems, Inc.

1. Loveless, A. (1969) Nature (London) 223, 206-207.

2. Pegg, A. E. \& Singer, B. (1984) Cancer Invest. 2, 223-236.

3. Demple, B., Jacobson, A., Olsson, M., Robins, P. \& Lindahl, T. (1982) J. Biol. Chem. 257, 13776-13780. 
4. Boulden, A. M., Foote, R. S., Fleming, G. S. \& Mitra, S. (1987) J. Biosci. 11, 215-224.

5. Bhattacharyya, D., Tano, K., Bunick, G. J., Uberbacher, E. C., Behnke, W. D. \& Mitra, S. (1988) Nucleic Acids Res. 16, 6397-6410.

6. Demple, B., Sedgwick, B., Robins, P., Totty, N., Waterfield, W. D. \& Lindahl, T. (1985) Proc. Natl. Acad. Sci. USA 82, 2688-2692.

7. Lindahl, T., Sedgwick, B., Sekiguchi, M. \& Nakabeppu, Y. (1988) Annu. Rev. Biochem. 57, 133-157.

8. Potter, P. M., Wilkinson, M. C., Fitton, J., Carr, F. J., Brennand, J., Cooper, D. P. \& Margison, G. P. (1987) Nucleic Acids Res. 15, 9177-9193.

9. Yarosh, D. B. (1985) Mutat. Res. 145, 1-16.

10. Foote, R. S. \& Mitra, S. (1984) Carcinogenesis 5, 277-281.

11. D'Ambrosio, S. M., Samuel, M. J., Dutta-Choudhury, T. A. \& Wani, A. A. (1987) Cancer Res. 47, 51-55.

12. Washington, W. J., Foote, R. S., Dunn, W. C., Generoso, W. M. \& Mitra, S. (1989) Mech. Ageing Dev. 48, 43-52.

13. Sagher, D., Karrison, T., Schwartz, J. L., Larson, R. A. \& Strauss, B. (1989) Cancer Res. 49, 5339-5344.

14. Russell, W. L., Hunsicker, P. R., Carpenter, D. A., Cornett, C. V. \& Guinn, G. M. (1982) Proc. Natl. Acad. Sci. USA 79, 3592-3593.

15. Day, R. S., III, Ziolkowski, C. H. J., Scudiero, D. A., Meyer, S. A., Lubiniecki, A. S., Giardi, A. J., Galloway, S. M. \& Bynum, G. D. (1980) Nature (London) 288, 724-727.

16. Sklar, R. \& Strauss, B. (1981) Nature (London) 289, 417-420.

17. Foote, R. S., Dunn, W. C. \& Mitra, S. (1986) in Environmental Mutagenesis and Carcinogenesis, eds. Notani, N. K. \& Chauhan, P. S. (Bhabha Atomic Res. Centre, Bombay, India), pp. 99-115.

18. Yarosh, D. B., Foote, R. S., Mitra, S. \& Day, R. S., III (1983) Carcinogenesis 4, 199-205.

19. Ayres, K., Sklar, M., Larson, K., Lindgren, V. \& Strauss, B. (1982) Mol. Cell. Biol. 2, 904-913.
20. Brent, T. P., Houghton, P. J. \& Houghton, J. A. (1985) Proc. Natl. Acad. Sci. USA 82, 2985-2989.

21. Schold, S. C., Jr., Brent, T. P., Von Hofe, E., Friedman, H. S., Mitra, S., Bigner, D. D., Swenberg, J. A. \& Kleihues, P. (1989) J. Neurosurg. 70, 573-577.

22. Ding, R., Ghosh, K., Eastman, A. \& Bresnick, E. (1985) Mol. Cell. Biol. 5, 3293-3296.

23. Dunn, W. C., Tano, K., Foote, R. S., Horesovsky, G. J., Preston, R. J. \& Mitra, S. (1988) J. Cell. Biochem., Suppl. 12A, 312 (abstr.).

24. Shevell, D. E., Abou-Zamzam, A. M., Demple, B. \& Walker, G. C. (1988) J. Bacteriol. 170, 3294-3296.

25. Yanisch-Perron, C., Vieira, J. \& Messing, J. (1985) Gene 33, 103-119.

26. Tano, K., Foote, R. S. \& Mitra, S. (1988) Gene 64, 305-311.

27. Maniatis, T., Fritsch, E. F. \& Sambrook, J. (1982) Molecular Cloning: A Laboratory Manual (Cold Spring Harbor Lab., Cold Spring Harbor, NY).

28. Gubler, U. \& Hoffman, B. J. (1983) Gene 25, 263-269.

29. Feinberg, A. P. \& Vogelstein, B. (1983) Anal. Biochem. 132, 6-13.

30. Sanger, F., Nicklen, S. \& Coulson, A. R. (1977) Proc. Natl. Acad. Sci. USA 74, 5463-5467.

31. Hattori, M. \& Sakaki, Y. (1986) Anal. Biochem. 152, 232-238.

32. Matsudaira, P. (1987) J. Biol. Chem. 262, 10035-10038.

33. Porter, M. A., Milanez, S., Stringer, C. D. \& Hartman, F. C. (1986) Arch. Biochem. Biophys. 245, 14-23.

34. Lipman, D. J. \& Pearson, W. R. (1985) Science 227, 14351441.

35. Fujimura, R. K., Valdivia, R. P. \& Allison, M. A. (1988) DNA Protein Eng. Tech. 1, 45-48.

36. Gunning, P., Ponte, P., Okayama, H., Engel, J., Blau, H. \& Kedes, L. (1983) Mol. Cell. Biol. 3, 787-795.

37. Bootsma, D., Westerveld, A. \& Hoeijmakers, J. H. J. (1988) Cancer Surv. 7, 303-315.

38. Weber, C. A., Salazar, E. P., Stewart, S. A. \& Thompson, L. H. (1988) Mol. Cell. Biol. 8, 1137-1146.

39. Doolittle, R. F. (1989) Trends Biochem. Sci. 14, 244-245. 